\title{
FURTHER RESULTS ON FIXPOINTS AND ZEROS OF ENTIRE FUNCTIONS
}

\author{
JIAN-HUA ZHENG AND CHUNG-CHUN YANG
}

\begin{abstract}
In this paper, a quantitative estimation on the number of zeros of the function $f \circ g(z)-\alpha(z)$ is derived, where $f$ and $g$ are transcendental entire functions and $\alpha(z)$ a nonconstant polynomial. As an application of this and a further step towards an affirmative answer to a conjecture of Baker, a quantitative estimation on the number of period points of exact order $n$ of $f_{n}$ ( $n$th iterate of $f$ ) is obtained.
\end{abstract}

\section{INTRODUCTION}

Let $f(z)$ be an entire function and $f_{n}(z)=f_{n-1}(f(z))\left(f_{1}(z) \equiv f(z), f_{0}(z)\right.$ $\equiv z$ ) be the $n$th iterate of $f$. A point $z_{0}$ at which $f\left(z_{0}\right)=z_{0}$ is called a fixpoint of $f(z)$. A fixpoint $z_{0}$ of order $k$ of $f(z)$ is a zero of $f_{k}(z)-z$ and, further, according to whether

$$
\left|f_{k}^{\prime}\left(z_{0}\right)\right|>1, \quad\left|f_{k}^{\prime}\left(z_{0}\right)\right|<1, \quad \text { or } \quad\left|f_{k}^{\prime}\left(z_{0}\right)\right|=1,
$$

it is called, respectively, repulsive, attractive, or indifferent, and such a $z_{0}$ is a fixpoint of exact order $k$ of $f(z)$ if it is not a fixpoint of order less than $k$ of $f(z)$.

Rosenbloom [15] seems to be the first to use the methods of Nevanlinna's theory to study the existence and number of fixpoints of transcendental entire functions. He showed that any transcendental entire function has infinitely many fixpoints of order 1 or 2. Baker [2], as a generalization, proved:

Theorem A. Any transcendental entire function $f(z)$ has infinitely many fixpoints of exact order $n(n \geq 2)$ with the exception of at most one value of $n$, where if $f(z)$ has some finite deficient value, cannot occur (cf. [3]).

Baker also raised

Conjecture (A). For $n \geq 2, f(z)$ must have infinitely many fixpoints of exact order $n$.

Recently, Bergweiler [6] completely answered the conjecture and obtained an improved result as follows:

Theorem B. Let $f(z)$ be an entire transcendental function $n \geq 2$. Then $f(z)$ has an infinite number of repulsive fixpoints of exact order $n$.

Received by the editors December 27, 1991.

1991 Mathematics Subject Classification. Primary 30D05, 30D20.

C)1995 American Mathematical Society 
There are a number of papers (cf. [4], [5], [8]) dealing with the existence of the fix-points of $f(g)$ for entire $f$ and $g$, but no significant quantitative estimation on the number of fix-points were obtained. In this paper, we shall present, by slightly modifying an important lemma of [4], a lower bound on the estimation of $N(r, 1 / f(g(z))-\alpha(z))$ (where $f$ and $g$ are transcendental entire functions and $\alpha$ is a nonconstant polynomial) and use this to obtain some results toward the answering of the following question raised by Baker [3] in 1960:

Question (A). Is $N_{n}(r)$ the same growth as $T\left(r, f_{n}\right)$, where $N_{n}(r)$ denotes the counting function for fixpoints of exact order $n$ of $f(z)$ ?

About this question, Baker [1] proved the following

Theorem C. Let the order $\rho(f)$ of the entire function $f$ be less than $1 / 2$ and

$$
f(z)-z=c_{p} z^{p}+c_{p+1} z^{p+1}+\cdots, \quad c_{p} \neq 0 .
$$

Then for sufficiently large $r$

$$
\log \left\{M\left(r, f_{n}\right)-r^{k}\right\}<\log \left|c_{p}\right|+p k \log r+N\left(r^{k}, \frac{1}{f_{n}-z}\right),
$$

where $k=k(n) \geq \frac{3(c+1) k(n-1)}{\cos \pi \rho}, k(1)=3(c+1), c>1$, and hence $k(n)>3^{n}$.

Throughout this paper, we shall use such standard notations as $N(r, 1 / f)$, $T(r, f), \delta(0, f)$, etc. of Nevanlinna theory and assume that the reader is familiar with its fundamental theorems, and by $\rho(f)$ and $\lambda(f)$ we denote the order and the lower order of $f(z)$, respectively, and by $E$ and $F$ sets of $r$ with, respectively, finite linear measure and finite logarithmic measure, respectively not necessarily the same at each occurrence. Finally, we recall that it was Rosenbloom [15] who proved that for a polynomial $P(z)$ and a transcendental entire function $g(z)$,

$$
\limsup _{r \rightarrow \infty} \frac{N(r, 1 /(P(g)-z))}{T(r, g)} \geq 1
$$

and conjectured the limsup is greater than or equal to $k-1$, where $k$ is the degree of $P(z)$. This was later confirmed by G. S. Prokopovich [14] by showing that the limsup is greater than $k-1+\delta(0, g)$ with $r \notin E$. Recently Bergweiler (private communication) suggested the lim sup, in general, is $k-2$ for $P(z)$ being a rational function. Here we shall present a lower bound for the number of distinct zeros of $P(g)-z$ in term of the degree of $P(z)$.

\section{Preliminary lemmas}

Lemma 1. Let $n(t)$ be a nonnegative, nondecreasing function and satisfy

$$
\limsup _{t \rightarrow \infty} \frac{\log n(t)}{\log t}=\infty
$$

Then there exists a set I of infinite logarithmic measure such that, for $r \in I$,

$$
(1+o(1)) \frac{\log n(r)}{\log r} \geq \sup \left\{\frac{\log n(t)}{\log t} ; e \leq t \leq r\right\} .
$$


Proof. Since

$$
\limsup _{t \rightarrow \infty} \frac{\log n(t)}{\log t}=\infty,
$$

there exists an unbounded sequence $\left\{r_{n}\right\}$ such that

$$
\frac{\log n\left(r_{n}\right)}{\log r_{n}}=\sup \left\{\frac{\log n(t)}{\log t} ; e \leq t \leq r_{n}\right\} \text {. }
$$

Then for $r_{n} \leq r \leq 2 r_{n}$,

$$
\begin{aligned}
\sup \left\{\frac{\log n(t)}{\log t} ; e \leq t \leq r\right\} & =\sup \left\{\frac{\log n(t)}{\log t} ; r_{n} \leq t \leq r\right\} \\
& \leq \frac{\log n(r)}{\log r_{n}}=\frac{\log n(r)}{\log r} \frac{\log r}{\log r_{n}}=(1+o(1)) \frac{\log n(r)}{\log r}
\end{aligned}
$$

Thus Lemma 1 follows.

Let $\left\{a_{n}\right\}$ be a sequence of complex numbers and $\left|a_{n}\right| \rightarrow \infty$ and let $r_{n}=\left|a_{n}\right|$ with $0<r_{1} \leq r_{2} \leq \cdots \leq r_{n} \leq \cdots$. Define

$$
\sigma_{n}=\frac{\log n}{\log r_{n}}, \quad n=r_{n}^{\sigma_{n}}
$$

and

$$
\rho(r)=\log n(r) / \log r, \quad n\left(r_{n}\right) \geq n,
$$

where $n(r)$ denotes the number of $\left\{a_{n}\right\}$ in $|z| \leq r$. Clearly

$$
\rho\left(r_{n}\right)=\log n\left(r_{n}\right) / \log r_{n} \geq \sigma_{n},
$$

and hence

$$
r_{n}^{\rho\left(r_{n}\right)} \geq n
$$

Define $p_{n}=\left[2 \rho\left(r_{n}\right)\right]$, where $[x]$ denotes the largest integer no greater than $x$. And obviously the sum

$$
\sum_{n=1}^{\infty}\left(r / r_{n}\right)^{p_{n}+1}
$$

converges for any $r>0$ and thus

$$
P(z)=\prod_{n=1}^{\infty} E\left(\frac{z}{z_{n}}, p_{n}\right)
$$

defines a transcendental entire function, where

$$
E(z, p)=(1-z) \exp \left(z+z^{2} / 2+\cdots+z^{p} / p\right) .
$$

Lemma 2. For all sufficiently large $r$, and $\varepsilon>0$,

$$
\log |P(z)|<n(r)^{2 \log r}+n\left(r^{2+\varepsilon} / 2\right)\left(3+\log n\left(r^{2+\varepsilon} / 2\right)\right) .
$$

And further if

$$
\limsup _{r \rightarrow \infty} \frac{\log n(r, 1 / P)}{\log r}=\infty,
$$

then there exists a set $I$ of infinite logarithmic measure such that, for $r \in I$,

$$
\log |P(z)|<n\left(r^{2+\varepsilon} / 2\right)^{7 / 2} \text {. }
$$

In order to prove the above lemma, we need the following 
Lemma $3([9$, p. 26]). For all $z$ and $p \geq 1$,

$$
\log |E(z, p)| \leq A(p) \min \left\{|z|^{p},|z|^{p+1}\right\},
$$

where $A(p)=2(2+\log p)$.

Proof of Lemma 2. Notice

$$
p_{n} \leq 2 \rho\left(r_{n}\right)=2 \log n\left(r_{n}\right) / \log r_{n}<p_{n}+1
$$

and when $r_{n} \geq \exp \left(e^{3}\right), A\left(p_{n}\right) \leq 2 \log \log n\left(r_{n}\right)$. Thus it follows from Lemma 3 that, for $|z|=r>\exp \left(e^{3}\right)$,

$$
\begin{aligned}
\log |P(z)| & =\sum_{n=1}^{\infty} \log \left|E\left(\frac{z}{z_{n}}, p_{n}\right)\right| \\
\leq & 2 \sum_{r \geq r_{n} \geq \exp \left(e^{3}\right)}\left(\log \log n\left(r_{n}\right)\right)\left(r / r_{n}\right)^{p_{n}}+\sum_{r<r_{n}}\left(\log \log n\left(r_{n}\right)\right)\left(r / r_{n}\right)^{p_{n}+1} \\
& +O\left(r^{n\left(\exp \left(e^{3}\right)\right)+1}\right) \\
< & \int_{e}^{\infty} 2(\log \log n(t))(r / t)^{2 \rho(t)} d n(t)+O\left(r^{n\left(\exp \left(e^{3}\right)\right)+1}\right) \\
= & 2\left\{\int_{e}^{r}+\int_{r}^{r^{2+\varepsilon} / 2}+\int_{r^{2+\varepsilon} / 2}^{\infty}\right\}(\log \log n(t))(r / t)^{2 \rho(t)} d n(t)+O\left(r^{n\left(\exp \left(e^{3}\right)\right)+1}\right)
\end{aligned}
$$

where $\varepsilon$ is a given positive number. We now express the three integrals above as I, II, and III, respectively and estimate them below.

We note when $r$ is sufficiently large, for $x>n\left(r^{2+\varepsilon} / 2\right)$,

$$
(\log \log x)<x^{(\varepsilon \log r+3 \log 2) / 2((2+\varepsilon) \log r-\log 2)} .
$$

$$
\begin{aligned}
\mathrm{III} & =\int_{r^{2+\varepsilon} / 2}^{\infty}(\log \log n(t)) n(t)^{-2(1-\log r / \log t)} d n(t) \\
& \leq \int_{r^{2+\varepsilon} / 2}^{\infty}(\log \log n(t)) n(t)^{-[1+(\varepsilon \log r+3 \log 2) /((2+\varepsilon) \log r-\log 2)]} d n(t) \\
& <\int_{n\left(r^{2+\varepsilon} / 2\right)}^{\infty}(x)^{-[1+(\varepsilon \log r+3 \log 2) / 2((2+\varepsilon) \log r-\log 2)]} d x<1 ; \\
\mathrm{II} & \leq \int_{r}^{r^{2+\varepsilon} / 2} \log \log n(t) d n(t) \\
& <n\left(r^{2+\varepsilon} / 2\right) \log \log n\left(r^{2+\varepsilon} / 2\right)-n(r) \log \log n(r)-1 ; \\
\mathrm{I} & <\log \log n(r) \int_{e}^{r}(r / e)^{2 \log n(t) / \log t} d n(t)<\log \log n(r) \int_{e}^{r} n(t)^{2(\log r-1)} d n(t) \\
& <\log \log n(r) n(r)^{2 \log r-1 .} .
\end{aligned}
$$

Combining I, II, and III we obtain the first inequality of Lemma 2.

As to the second inequality, we only need to improve the estimate of integral I. By Lemma 1, there exists a set $I$ of infinite logarithmic measure such that for $r \in I$

$$
(1+o(1)) \frac{\log n(r)}{\log r} \geq \sup \left\{\frac{\log n(t)}{\log t} ; e \leq t \leq r\right\} .
$$


Therefore for $r \in I$

$$
\begin{aligned}
\mathrm{I} & <\log \log n(r) \int_{e}^{r}(r / e)^{2 \log n(t) / \log t} d n(t) \\
& <\log \log n(r) \int_{e}^{r}(r / e)^{(1+o(1)) 2 \log n(r) / \log r} d n(t) \\
& =\log \log n(r) n(r)^{2(1+o(1))+1}<n(r)^{3+1 / 2} .
\end{aligned}
$$

This also completes the proof of Lemma 2.

Lemma 4 ([7] or cf. [4, p. 76]). If $f$ and $g$ are transcendental entire functions, then

$$
M(r, f(g))=M((1+o(1)) M(r, g), f) \quad(r \notin F) .
$$

The following results in essence come from Wiman-Valiron theory. We first denote the central index of an entire function $g(z)$ by $\nu(r, g)$ and the maximum term by $\mu(r, g)$.

Lemma 5 ([16] or cf. [4]). Let $g$ be entire and transcendental and assume that $d>0, \varepsilon>0, \eta>0$, and $\gamma>1 / 2$. Suppose that $\left|z_{0}\right|=r,\left|g\left(z_{0}\right)\right| \geq$ $\eta M(r, g)$, and $|\tau|<d \nu(r, g)^{-\gamma}$. Then

(5) $g\left(z_{0} e^{\tau}\right) \sim g\left(z_{0}\right) e^{\nu(r, g) \tau} \quad(r \notin F)$;

(6) $g^{\prime}\left(z_{0} e^{\tau}\right) \sim\left(\nu(r, g) / z_{0} e^{\tau}\right) g\left(z_{0}\right) e^{\nu(r, g) \tau} \quad(r \notin F)$;

(7) $\nu(r, g) \leq(\log \mu(r, g))^{1+\varepsilon} \leq(\log M(r, g))^{1+\varepsilon} \quad(r \notin F)$;

(8) $\log M(r, g) \leq(1+o(1)) \log \mu(r, g) \leq(1+o(1)) \nu(r, g) \log r \quad(r \notin F)$.

By suitably modifying a proof of Bergweiler [4, Lemma 3], we can easily derive the following

Lemma 6. Let $g(z)$ be entire and transcendental and assume that $c>0,1 \geq$ $\gamma>1 / 2$, and $\eta>0$. If $r \notin F,\left|z_{0}\right|=r$, and $\left|g\left(z_{0}\right)\right| \geq \eta M(r, g)$, then there exists a function $\tau(z)$ analytic in $\left|z-z_{0}\right| \leq \operatorname{cr\nu }(r, g)^{-\gamma}$ satisfying

$$
|\tau(z) \nu(r, g)-2 \pi i|=o(1), \quad g\left(z e^{\tau(z)}\right)=g(z) .
$$

Let $f(z)$ and $g(z)$ be two transcendental entire functions and $\alpha(z)$ a nonconstant polynomial. By $\beta(z)$ we denote the Weierstrass product of the zeros of $f(g)-\alpha(z)$ constructed in the same manner as $P(z)$ in (2). We note if $f(g)-\alpha(z)$ has the finite exponent of convergence of the zeros, $\beta(z)$ must be of finite order. Thus we have

$$
f(g(z))=\alpha(z)+\beta(z) e^{D(z)}
$$

for some entire function $D(z)$.

Lemma 7. If $g$ and $\beta$ are defined as in the paragraph above and satisfy the relationship

$$
N(r, 1 / \beta(z))<d T\left(r^{1 / 3}, g\right) \quad(r \notin F) ;
$$

where $d$ is a given number, $0<d<1$, then there exists a set $I$ of infinite logarithmic measure such that for $r \in I$,

$$
\nu(r, g)<\nu(r, D)^{1+\varepsilon},
$$


where $\varepsilon$ is a given positive number. Further when $\lambda(g)>0, I=R \backslash F$.

Proof. First suppose that the derivative $f^{\prime}(z)$ has only a finite number of zeros. Then

$$
\begin{aligned}
\bar{N}\left(r, \frac{1}{(f(g))^{\prime}}\right) & \leq \bar{N}\left(r, \frac{1}{f^{\prime}(g)}\right)+\bar{N}\left(r, \frac{1}{g^{\prime}}\right)=O(T(r, g)) \\
& =o\left(T\left(r,(f(g))^{\prime}\right)\right) \quad(r \notin E)
\end{aligned}
$$

$$
\begin{aligned}
\bar{N}(r & \left., \frac{1}{(f(g))^{\prime}-\alpha^{\prime}}\right) \\
& =\bar{N}\left(r, \frac{1}{\beta^{\prime}+\beta D^{\prime}}\right)<\bar{N}\left(r, \frac{1}{\beta}\right)+T\left(r, \frac{\beta^{\prime}}{\beta}+D^{\prime}\right)+O(1) \\
& =2 \bar{N}\left(r, \frac{1}{\beta}\right)+m\left(r, \frac{(f(g)-\alpha)^{\prime}}{f(g)-\alpha}\right)+O(1)=o\left(T\left(r,(f(g))^{\prime}\right)\right) \quad(r \notin E) .
\end{aligned}
$$

These two inequalities will lead to an absurdity. Hence $f^{\prime}(z)$ has infinitely many zeros. Differentiating both sides of (9), we have

$$
f^{\prime}(g(z)) g^{\prime}(z)=\left(\beta^{\prime}(z) / \beta(z)+D^{\prime}(z)\right)(f(g(z))-\alpha(z))+\alpha^{\prime}(z) .
$$

If $f^{\prime}(a)=0$, then

$$
\begin{aligned}
N\left(r, \frac{1}{g-a}\right) & \leq N\left(r, \frac{1}{\left(\beta^{\prime} / \beta+D^{\prime}\right)(f(a)-\alpha)+\alpha^{\prime}}\right) \\
& \leq T\left(r,\left(\beta^{\prime} / \beta+D^{\prime}\right)(f(a)-\alpha)+\alpha^{\prime}\right)+O(1) \\
& \leq T\left(r, D^{\prime}\right)+T\left(r, \beta^{\prime} / \beta\right)+o(T(r, g)) \\
& =T\left(r, D^{\prime}\right)+\bar{N}(r, 1 / \beta)+m\left(r, \beta^{\prime} / \beta\right)+o(T(r, g)) \\
& \leq(1+o(1)) T(r, D)+d T(r, g)+m\left(r, \beta^{\prime} / \beta\right)+o(T(r, g)) .
\end{aligned}
$$

Now we estimate $m\left(r, \beta^{\prime} / \beta\right)$ by Lemma 2 and the well-known lemma of logarithmic derivative.

$$
\begin{aligned}
m\left(r, \beta^{\prime} / \beta\right) & <O(\log T(r, \beta)) \leq O(\log \log M(r, \beta)) \\
& <O\left(\log r \log n(r, 1 / \beta)+\log n\left(r^{5 / 2} / 2,1 / \beta\right)\right) \\
& =O\left(n\left(r^{5 / 2} / 2,1 / \beta\right)\right)
\end{aligned}
$$

and

$$
n\left(r^{5 / 2} / 2,1 / \beta\right)<(\log 2)^{-1} N\left(r^{5 / 2} / 2,1 / \beta\right)<(d / \log 2) T(r, g) .
$$

Hence

$$
m\left(r, \beta^{\prime} / \beta\right)=o(T(r, g)) .
$$

Since $f^{\prime}(z)$ has infinitely many zeros, from Nevanlinna's second fundamental theorem, (12) and (13) it follows that

$$
T(r, g) \leq O(T(r, D)) \quad(r \notin E) .
$$

A classical lemma due to Borel [9, Lemma 2.4] tells us that

$$
\log M(r, g)<T(r, g)^{1+\varepsilon / 6} \quad(r \notin F) .
$$


Combining (7), (15), (14), and (8), we obtain

$$
\begin{aligned}
\nu(r, g) & \leq(\log M(r, g))^{1+\varepsilon / 6} \leq T(r, g)^{1+\varepsilon / 3}<T(r, D)^{1+\varepsilon / 2} \\
& \leq(\log M(r, D))^{1+\varepsilon / 2} \leq(\nu(r, D) \log r)^{1+5 \varepsilon / 6} \quad(r \notin F) .
\end{aligned}
$$

Thus the same argument as in W. Bergweiler [4] implies that there exists a set $I$ of $r$ of infinite logarithmic measure such that for $r \in I$,

$$
\nu(r, g) \leq \nu(r, D)^{1+\varepsilon} \text {. }
$$

Further it follows from (16) that when the lower order of $g$ is positive,

$$
\nu(r, g) \leq \nu(r, D)^{1+\varepsilon} \quad(r \notin F) .
$$

This completes the proof of Lemma 7.

Lemma $8([17$, p. 68]). If $f(z)$ is meromorphic in $|z|<R$, with $f(0) \neq 0,1, \infty$ and $f^{\prime}(0) \neq 0$, then for $0<r<R$,

$$
\begin{aligned}
T(r, f)< & 2\left\{N(R, f)+N\left(R, \frac{1}{f}\right)+N\left(R, \frac{1}{f-1}\right)\right\} \\
& +191+4 \log ^{+}|f(0)|+2 \log ^{+} \frac{1}{R|f(0)|}+12 \log ^{+} \frac{R}{R-r} .
\end{aligned}
$$

The following is a classical inequality of Nevanlinna, which can be found in $[11$, p. 120$]$ or $[10$, p. 331$]$.

Lemma 9. Suppose that $h_{0}(z), \ldots, h_{p}(z)$ are linearly independent meromorphic functions and not all rational, and such that

$$
h_{0}(z)+\cdots+h_{p}(z) \equiv 1 \text {. }
$$

Then

$$
T\left(r, h_{\nu}\right)<p\left\{\sum_{k=0}^{p} \bar{N}\left(r, \frac{1}{h_{k}}\right)+\sum_{k \neq \nu} \bar{N}\left(r, h_{k}\right)\right\}+S(r),
$$

where

$$
S(r)=O\left(\log \left(\sum_{k=0}^{p} T\left(r, h_{k}\right)\right)\right)+O(\log r), \quad \text { as } r \rightarrow \infty, r \notin E .
$$

The following result is obvious.

Lemma 10. Let

$$
P(z)=a_{s} z^{s}+a_{s-1} z^{s-1}+\cdots+a_{0} \quad\left(a_{s} \neq 0 ; s \geq 1\right) .
$$

Then for any meromorphic function $f(z)$, we have

$$
T(r, P(f))<s T(r, f)+s \log 2+\sum_{k=0}^{s} \log ^{+}\left|a_{k}\right| .
$$

\section{STATEMENTS OF MAIN RESULTS}

Theorem 1. Let $f$ and $g$ be two transcendental entire functions and $\alpha(z) a$ nonconstant polynomial. If $\lambda(f)>0$, and $g(z)$ satisfies

$$
(\log r)^{3}=O(\nu(r, g)),
$$


then there is a set I of infinite logarithmic measure such that for $r \in I$, we have

$$
N\left(r, \frac{1}{f(g)-\alpha}\right)>\left[\log M\left(r^{1 / 3}, f(g)\right)\right]^{1 / 4} \text {. }
$$

Theorem 2. If $f(z), g(z)$ are defined as in Theorem 1, then there exists a set $I$ of infinite logarithmic measure such that for $r \in I$,

$$
N\left(r, \frac{1}{f(g)-\alpha}\right)>\min \left\{d T\left(r^{1 / 3}, g\right),\left[\log M\left(r^{1 / 3}, f(g)\right)\right]^{1 / \log r}\right\} .
$$

Furthermore when $\lambda(g)>0, I=R \backslash F$.

The following result can be derived immediately from Theorem 1 , which is related to Question (A).

Theorem 3. If $f(z)$ is a transcendental entire function with $\lambda(f)>0$, then there exists a set $I$ of $r$ of infinite logarithmic measure such that for $r \in I$,

$$
N_{n}(r)>(1-o(1))\left[\log M\left(r^{1 / 3}, f_{n}\right)\right]^{1 / 4} \quad(n>2) .
$$

Theorem 4. Let $P(z)$ be a nonconstant polynomial and $f(z)$ a transcendental entire function and $\alpha(z)$ a nonconstant small meromorphic function satisfying $T(r, \alpha(z))=o(T(r, f))$. Then $P(f(z))-\alpha(z)$ must have an infinite number of zeros and furthermore

$$
T(r, P(f))<k \bar{N}\left(r, \frac{1}{P(f)-\alpha}\right)+o(T(r, f)), \quad r \notin E .
$$

where $k=2$ if $P^{\prime}(z)$ has only one zero; otherwise $k=2 \operatorname{deg} P$.

From Theorem 4, we may immediately get a corollary which provides a solution to Question (A).

Corollary. If a transcendental entire function $f(z)$ has the factorization $f(z)=$ $P(g(z))$ for some nonlinear polynomial $P(w)$ and entire function $g(z)$, then for sufficiently large $r$,

$$
N_{n}\left(r, 1 /\left(f_{n}-z\right)\right)>k T\left(r, f_{n}\right), \quad r \notin E,
$$

where $k$ is a positive number dependent on the $\operatorname{deg} P$.

\section{Proof of Theorem 1}

First we write

$$
f(g(z))=\alpha(z)+\beta(z) e^{D(z)},
$$

where $D(z)$ is an entire function and $\beta(z)$ a Weierstrass product formed by the zeros of $f(g)-\alpha(z)$ as expressed in Lemma 2.

We need treat two cases, separately.

(I) $40 \log M(2 r, \beta(z)) \geq \log M(r, f(g)), r \notin F$. Obviously

$$
\limsup _{r \rightarrow \infty} \frac{\log n(r, 1 / \beta)}{\log r}=\infty .
$$

Otherwise, the order of $\beta(z)$ is finite, but on the other hand, the lower order of $f(g)$ is infinite and hence $\log M(2 r, \beta(z))=o(\log M(r, f(g)))$, which is 
a contradiction. Thus it follows from Lemma 2 that there exists a set $I$ of infinite logarithmic measure such that $r \in I$,

$$
\log M(r, f(g)) \leq 40 n\left(r^{2+\varepsilon}\right)^{7 / 2} .
$$

Thus we easily get Theorem 2 .

(II) There exists a set $I$ of infinite logarithmic measure such that $r \in I$,

$$
40 \log M(2 r, \beta(z))<\log M(r, f(g)) \text {. }
$$

Suppose now that the theorem is false; then

$$
N\left(r \frac{1}{f(g)-\alpha}\right) \leq\left[\log M\left(r^{1 / 3}, f(g)\right)\right]^{1 / 4} .
$$

Obviously

$$
N\left(2 r, \frac{1}{f(g)-\alpha(z)}\right)=o(\log M(r, f(g))) .
$$

Since $\lambda(f)>0$, there exists a positive number $t$ such that $\log M(r, f) \geq r^{t}$, $r \notin E$. Thus by Lemma 4 , we have

$$
\log M(r, f(g))>(1-o(1))^{t} M(r, g)^{t} .
$$

On the other hand, we have

$$
\begin{aligned}
\log M(r, f(g)) & =\log M\left(r, \alpha(z)+\beta(z) e^{D(z)}\right) \\
& \leq O(\log r)+\log 2+\log M(r, \beta)+\log M\left(r, e^{D(z)}\right) \\
& \leq \frac{1}{20} \log M(r, f(g))+M(r, D(z)) .
\end{aligned}
$$

Thus

$$
\log M(r, D) \geq(t / 2) \log M(r, g) .
$$

It follows from this that for sufficiently large $r$,

$$
\nu(r, g)<(\log M(r, g))^{7 / 6}<((2 / t) \log M(r, D))^{7 / 6} \leq((3 / t) \nu(r, D) \log r)^{7 / 6} \text {. }
$$

Combining the above inequalities and the assumption that for some positive number $c,(\log r)^{3} \leq c \nu(r, g)$, we have

$$
\nu(r, g)<(3 / t)^{21 / 11} \nu(r, D)^{21 / 11}<\nu(r, D)^{23 / 12} .
$$

We choose a point $z_{0}$ with $\left|z_{0}\right|=r$ such that

$$
\left|f\left(g\left(z_{0}\right)\right)\right|=M(r, f(g))=M((1+o(1)) M(r, g), f) ;
$$

then

$$
\left|g\left(z_{0}\right)\right| \geq(1-o(1)) M(r, g),
$$

and

$$
\begin{aligned}
\left|D\left(z_{0}\right)\right| & >\operatorname{Re} D\left(z_{0}\right)=\log \left|\exp \left(D\left(z_{0}\right)\right)\right|=\log \left|\left(f\left(g\left(z_{0}\right)\right)-\alpha\left(z_{0}\right)\right) / \beta\left(z_{0}\right)\right| \\
& \geq \log \left|f\left(g\left(z_{0}\right)\right)\right|-\log ^{+}\left|\alpha\left(z_{0}\right)\right|-\log ^{+}\left|\beta\left(z_{0}\right)\right|-\log 2 \\
& \geq \log M(r, f(g))-\log M(r, \alpha(z))-\log M(r, \beta(z))-\log 2 \\
& \geq \log M(r, \exp (D(z)))-2\{\log M(r, \alpha(z))+\log M(r, \beta(z))+\log 2\} \\
& \geq(7 / 8)(1-o(1)) \log M(r, \exp (D(z)))>(2 / 3) M(r, D), \quad r \notin F .
\end{aligned}
$$


From Lemma 6, we can construct an analytic function $\tau(z)$ in $\left|z-z_{0}\right| \leq$ $12 c r \nu(r, g)^{-12 / 23}, c=\arctan 2+\pi / 2, r \notin E$, satisfying

$$
|\tau(z) \nu(r, g)-2 \pi i|=o(1), \quad \text { and } \quad g\left(z e^{\tau(z)}\right)=g(z) .
$$

Set $k(z)=z e^{\tau(z)}$ and

$$
h(z)=\frac{f(g(z))-\alpha(z)}{\alpha(k(z))-\alpha(z)}
$$

Since, as $r \rightarrow \infty$,

$$
\alpha(k(z))-\alpha(z) \sim\left(e^{q \tau(z)}-1\right) \alpha(z) \sim q \tau(z) \alpha(z) \sim(2 \pi q i / \nu(r, g)) \alpha(z),
$$

where $q$ is the degree of $\alpha(z)$, it follows that $h(z)$ is analytic in $\left|z-z_{0}\right| \leq$ $12 c r \nu(r, g)^{-12 / 23}$.

Now define $z_{1}=z_{0} e^{i \theta}$ where $\theta \in R$ is chosen such that $\operatorname{Re} D\left(z_{1}\right)=0$ and $|\nu(r, D) \theta-\pi / 2|=\left|\arg D\left(z_{0}\right)+o(1)\right|=\left|\arctan \left(\operatorname{Im} D\left(z_{0}\right) / \operatorname{Re} D\left(z_{0}\right)\right)+o(1)\right|<$ $\arctan 2$, we can do this by Lemma 5 and (21). Then

$$
\left|z_{1}-z_{0}\right|=r\left|e^{i \theta}-1\right| \sim r|\theta|<\operatorname{cr\nu }(r, D)^{-1} .
$$

Since

$$
\nu(r, g)<\nu(r, D)^{23 / 12}, \quad \text { i.e. } \quad \nu(r, D)^{-1}<\nu(r, g)^{-12 / 23}, \quad r \notin F,
$$

we have $\left|z_{1}-z_{0}\right|<(1+o(1)) \operatorname{cr} \nu(r, g)^{-12 / 23}<2 c r \nu(r, g)^{-12 / 23}$.

It follows that $h(z)$ is well defined for $\left|z-z_{1}\right|<10 \operatorname{cr} \nu(r, g)^{-12 / 23}$ and, setting $R=2 \operatorname{cr} \nu(r, g)^{-12 / 23}$, we now estimate

$$
N(R)=N\left(5 R, z_{1}, h\right)+N\left(5 R, z_{1}, 1 / h\right)+N\left(5 R, z_{1}, 1 /(h-1)\right) .
$$

For this, we consider the disk $\left|z-z_{1}\right|<5 R$,

$$
\begin{aligned}
|z|<\left|z_{1}\right| & +5 R<\left|z_{0}\right|+6 R=r\left(1+12 c r \nu(r, g)^{-12 / 23}\right) \\
|k(z)| & =\left|z e^{\tau(z)}\right|<\left(1+12 c r \nu(r, g)^{-12 / 23}\right) r e^{|\tau(z)|} \\
< & \left(1+12 c r \nu(r, g)^{-12 / 23}\right)\left(1+7 c r \nu(r, g)^{-1}\right) r \\
< & \left(1+20 c r \nu(r, g)^{-12 / 23}\right) r, \quad r \notin F,
\end{aligned}
$$

since $|\tau(z) \nu(r, g)-2 \pi i|=o(1)$. Define $t=\left(1+20 c \nu(r, g)^{-12 / 23}\right) r$. Thus it clearly follows from $g(k(z))=g(z)$ and the form of $h(z)$ that

$$
N(R)<2 N(t, 1 / \beta)=o(\log M(r, f(g))), \quad r \in I \backslash F .
$$

Now we estimate $\log \left|h^{\prime}\left(z_{1}\right)\right|$ and $\log ^{+}\left|h\left(z_{1}\right)\right|$.

$$
\begin{aligned}
& \log ^{+}\left|h\left(z_{1}\right)\right|=\log ^{+}\left|\beta\left(z_{1}\right) \exp D\left(z_{1}\right) /\left(\alpha\left(k\left(z_{1}\right)\right)-\alpha\left(z_{1}\right)\right)\right| \\
& <\log ^{+}\left|\beta\left(z_{1}\right)\right|+\log ^{+}\left|\alpha\left(z_{1}\right)\right|+\log \nu(r, g)+O(1) \\
& <\log M(r, \beta(z))+O(\log r \nu(r, g)) \\
& <(1 / 40) \log M(r, f(g))+O(\log r \nu(r, g)) . \\
& h^{\prime}(z)=\frac{f^{\prime}(g(z)) g^{\prime}(z)-\alpha^{\prime}(z)}{\alpha(k(z))-\alpha(z)}-\frac{(f(g(z))-\alpha(z))\left(\alpha^{\prime}(k(z)) k^{\prime}(z)-\alpha^{\prime}(z)\right)}{(\alpha(k(z))-\alpha(z))^{2}} \\
& =\left[\frac{\beta^{\prime}(z)+\beta(z) D^{\prime}(z)}{\alpha(k(z))-\alpha(z)}-\frac{\beta(z)\left(\alpha^{\prime}(k(z)) k^{\prime}(z)-\alpha^{\prime}(z)\right)}{(\alpha(k(z))-\alpha(z))^{2}}\right] e^{D(z)} .
\end{aligned}
$$


Then (5) in Lemma 5 implies that

$$
\begin{aligned}
\log \left|h^{\prime}\left(z_{1}\right)\right|> & \log \left|D^{\prime}\left(z_{1}\right)\right|-\left[2 \log ^{+}\left|\beta\left(z_{1}\right)\right|+\log ^{+}\left|\beta^{\prime}\left(z_{1}\right)\right|+O(\log r \nu(r, g))\right] \\
> & \log M(r, D)+\log \nu(r, D)+O(1) \\
& -\left[2 \log M(r, \beta(z))+\log M\left(r, \beta^{\prime}(z)\right)+O(\log r \nu(r, g))\right], \\
\log M\left(r, \beta^{\prime}(z)\right) & <5 T\left(3 r / 2, \beta^{\prime}(z)\right)<5(1+o(1)) T(3 r / 2, \beta(z)) \\
& <(1 / 8) \log M(r, f(g)), \quad r \notin E .
\end{aligned}
$$

Thus

$$
\log \left|h^{\prime}\left(z_{1}\right)\right|>-(1 / 5) \log M(r, f(g)), \quad r \notin F .
$$

Now it follows from Lemma 8 that

$$
\begin{aligned}
T\left(4 R, z_{1}, h\right)< & 2 N(R)+191+12 \log ^{+} 5 R /(5 R-4 R) \\
& +2 \log ^{+} 1 / R+4 \log ^{+}\left|h\left(z_{1}\right)\right|+2 \log ^{+} 1 /\left|h^{\prime}\left(z_{1}\right)\right| \\
< & o(\log M(r, f(g)))+(1 / 2) \log M(r, f(g)) \\
& +O(\log r \nu(r, g)), \quad r \in I \backslash F .
\end{aligned}
$$

On the other hand, we have

$$
\left|h\left(z_{0}\right)\right|=\left|\frac{f\left(g\left(z_{0}\right)\right)-\alpha\left(z_{0}\right)}{\alpha\left(k\left(z_{0}\right)\right)-\alpha\left(z_{0}\right)}\right| \sim \frac{\nu(r, g)}{2 \pi q\left|\alpha\left(z_{0}\right)\right|}\left|f\left(g\left(z_{0}\right)\right)-\alpha\left(z_{0}\right)\right|,
$$

i.e.,

$$
\log \left|h\left(z_{0}\right)\right|>\log M(r, f(g))+\log \nu(r, g)-O(\log r)
$$

and

$$
\log \left|h\left(z_{0}\right)\right|<\log M\left(R, z_{1}, h\right) \leq(5 / 3) T\left(4 R, z_{1}, h\right) .
$$

Thus by combining (27), (28), and (29), we have $\log M(r, f(g))+\log \nu(r, g)$

$$
<o(\log M(r, f(g)))+O(\log r \nu(r, g))+(5 / 6) \log M(r, f(g)), \quad r \in I \backslash F,
$$

and hence

$$
T(r, f(g))<\log M(r, f(g))<O(\log r \nu(r, g)), \quad r \in I \backslash F .
$$

This is absurd and thus Theorem 1 follows.

\section{Proof of Theorem 2}

First we write

$$
f(g(z))=\alpha(z)+\beta(z) e^{D(z)},
$$

where $D(z)$ is an entire function and $\beta(z)$ a Weierstrass product formed by the zeros of $f(g)-\alpha(z)$ as expressed in Lemma 2 .

We need to treat two cases, separately.

(I) There exists a set $I$ of infinite logarithmic measure such that for $r \in I$,

$$
40 \log M(2 r, \beta(z)) \geq \log M(r / 2, f(g)) .
$$

Then Lemma 2 implies that

$$
\log M(r / 2, f(g))<40 n(r)^{2 \log r}+40 n\left(r^{5 / 2}\right) \log \log n\left(r^{5 / 2}\right)<41 n\left(r^{5 / 2}\right)^{2 \log r},
$$


and further

$$
\log M\left(r^{1 / 3}, f(g)\right)<41 n(r)^{(2 / 3) \log r}<n(r)^{2 \log r},
$$

this is (18).

(II)

$$
40 \log M(2 r, \beta(z))<\log M(r, f(g)), \quad r \notin F .
$$

Suppose now that the theorem is false so that

$$
N(r, 1 /(f(g)-\alpha(z)))<d T\left(r^{1 / 3}, g\right) \quad(0<d<1), r \notin F .
$$

From Lemma 7, for $0<d<1$, we can find a set $I$ of $r$ of infinite logarithmic measure such that $r \in I$,

$$
\nu(r, g)<\nu(r, D)^{1+\varepsilon},
$$

where $\varepsilon$ is a given positive number.

Then Theorem 2 follows by suitably modifying the proof of Case (II) of Theorem 1.

\section{Proof of Theorem 3}

Since $\lambda(f)>0$, Theorem 1 shows that there exists a set $I$ of infinite logarithmic measure such that for $r \in I$,

$$
N\left(r, \frac{1}{f_{n}-z}\right)>\left(\log M\left(r^{1 / 3}, f_{n}\right)\right)^{1 / 4}
$$

Let $z_{0}$ be a periodic point of order $n$, but not exact order $n$; i.e., there exists a positive integer $k(<n)$ such that

$$
f_{n}\left(z_{0}\right)=z_{0} \text { and } f_{k}\left(z_{0}\right)=z_{0} \text {, but } f_{j}\left(z_{0}\right) \neq z_{0} \text { for } j<k \text {. }
$$

Then $k \leq n-2$ and $n=m k$ for some integer $m>1$. Indeed, if $k=n-1$, then $f_{1}\left(z_{0}\right)=z_{0}$, and this will yield $n=2$, which is a contradiction. Now we can write for some positive integer $p$,

$$
f_{k}(z)-z_{0}=\left(z-z_{0}\right)^{p} f^{*}(z), \quad f^{*}\left(z_{0}\right) \neq 0 .
$$

Therefore we easily see

$$
f_{n}(z)-z_{0}=\left(z-z_{0}\right)^{p^{m}} f^{* p^{m-1}}(z) \cdots f^{* p}\left(f_{(m-2) k}(z)\right) f^{*}\left(f_{(m-1) k}(z)\right)
$$

so that

$$
N\left(r, \frac{1}{f_{n}-z}\right) \leq N_{n}(r)+\sum_{k=1}^{n-2} N_{k}^{n / k}(r) \leq N_{n}(r)+(1+o(1)) T^{n}\left(r, f_{n-2}\right) .
$$

On the other hand, we have

$$
\begin{aligned}
\log \log M\left(r^{1 / 3}, f_{n}\right) & \geq \log \log M\left(c M\left(r^{1 / 3} / 4, f\right), f_{n-1}\right)+O(1) \\
& \geq \log \log M\left(2 r, f_{n-1}\right)+O(1) \\
& \geq \log \log M\left(c M\left(r, f_{n-2}\right), f\right)+O(1) \\
& \geq \log M\left(r, f_{n-2}\right)+O(1) \geq T\left(r, f_{n-2}\right)+O(1),
\end{aligned}
$$

so that

$$
T^{n}\left(r, f_{n-2}\right)=o\left(\log M\left(r^{1 / 3}, f_{n}\right)^{1 / 4}\right) .
$$

Thus Theorem 3 follows. 


\section{Proof of Theorem 4}

Define $A(z)=P(f(z))-h(z)$; i.e.,

$$
P(f(z))=h(z)+A(z),
$$

and hence

$$
P^{\prime}(f(z)) f^{\prime}(z)=h^{\prime}(z)+A^{\prime}(z)=h^{\prime}(z)+\left(A^{\prime}(z) / A(z)\right)(P(f(z))-h(z)) .
$$

Below we treat two cases, separately.

(I) $P^{\prime}(z)$ has two distinct zeros, say $a_{i}(i=1,2)$. It follows from (31) that

$$
\begin{aligned}
& N\left(r, \frac{1}{f-a_{i}}\right) \leq N\left(r, \frac{1}{h^{\prime}(z)+\frac{A^{\prime}(z)}{A(z)}\left(P\left(a_{i}\right)-h(z)\right)}\right) \\
& \quad \leq \bar{N}\left(r, \frac{1}{P(f)-h}\right)+T\left(r, h^{\prime}\right)+T(r, h)+m\left(r, \frac{(P(f)-h)^{\prime}}{P(f)-h}\right)+O(1) \\
& \quad \leq \bar{N}\left(r, \frac{1}{P(f)-h}\right)+o(T(r, f)) .
\end{aligned}
$$

By Nevanlinna's second fundamental theorem and (32), one get

$$
\begin{aligned}
T(r, f) & <\sum_{i=1}^{2} N\left(r, \frac{1}{f-a_{i}}\right)+o(T(r, f)) \\
& <2 \bar{N}\left(r, \frac{1}{P(f)-h}\right)+o(T(r, f)) \quad(r \notin E) .
\end{aligned}
$$

This and Lemma 10 imply that

$$
T(r, P(f))<2 \operatorname{deg} P \bar{N}\left(r, \frac{1}{P(f)-h}\right)+o(T(r, f)) \quad(r \notin E) .
$$

(II) $P^{\prime}(z)$ has only one zero, say $a$. Then we can write $P^{\prime}(z)=n c(z-a)^{n-1}$, $n=\operatorname{deg} P, c$ is a constant; i.e., $P(z)=c(z-a)^{n}+b$, where $b$ is also a constant. It follows from (30) that $c(f(z)-a)^{n}+b=h(z)+A(z)$; that is,

$$
\frac{c(f(z)-a)^{n}}{h(z)-b}-\frac{A(z)}{h(z)-b}=1 \text {. }
$$

Applying Lemma 9 to the above equality, we have

$$
T\left(r, \frac{c(f(z)-a)^{n}}{h-b}\right)<\bar{N}\left(r, \frac{1}{f-a}\right)+\bar{N}\left(r, \frac{1}{A}\right)+o(T(r, f)), \quad r \notin E .
$$

Since $n \geq 2$ and $a$ is a zero of $P^{\prime}(z),(32)$ holds for $a$. This and the above inequality yield

$$
\begin{aligned}
T(r, P(f)) & <T\left(r, \frac{c(f-a)^{n}}{h-b}\right)+o(T(r, f)) \\
& <2 \bar{N}\left(r, \frac{1}{P(f)-h}\right)+o(T(r, f)) \quad(r \notin E) .
\end{aligned}
$$

Thus Theorem 4 is proved. 


\section{ConClusion}

Actually, Theorem 1 still holds, even if " $1 / 3$ " and " $1 / 4$ " there are respectively replaced by “ $\frac{1}{2+\varepsilon}$ " and “ $\frac{1}{3+\varepsilon}$ " ( $\varepsilon$ is a sufficiently small positive number). Indeed, one can easily verify this by analysing the proof of Theorem 1 . Also, under the hypothesis of Theorem 1, we can obtain the following result:

$$
N\left(r, \frac{1}{f(g)-\alpha}\right) \geq k \log M(r / 2, f(g)), \quad r \in I,
$$

where $0<k<1$ and $I$ has the positive lower logarithmic density, if there exist entire functions $\beta$ and $D$ such that

$$
f(g)-\alpha=\beta e^{D},
$$

with the properties that

(i) $T(r, \beta)<O\left(N\left(r, \frac{1}{\beta}\right)\right)$;

(ii) for some positive number $a, \log M(r, D) \geq a \log M(r, g)$, or $\lambda(f)>0$ and $(\log r)^{3}=O(\nu(r, g))$.

\section{REFERENCES}

1. I. N. Baker, Zusammensetzungen ganzer funktionen, Math. Z. 69 (1958), 121-163.

2. __ Fixpoints and iterates of entire functions, Math. Z. 71 (1959), 146-153.

3. _ The existence of fixpoints of entire functions, Math. Z. 73 (1960), 280-284.

4. W. Bergweiler, Proof of a conjecture of Gross concerning fixpoints, Math. Z. 204 (1990), 381-390.

5. _ On the fix-points of composite functions, Pacific J. Math. 143 (1990), 1-8.

6. __ Periodic points of entire functions: Proof of a conjecture of Baker (to appear).

7. J. Clunie, The composition of entire and meromorphic functions, Mathematical Essays Dedicated to A. J. Macintyre, Ohio Univ. Press, Athens, 1970, pp. 75-92.

8. F. Gross and C. Osgood, On fixed points of composite entire functions, J. London Math. Soc. (2) 28 (1983), 37-61.

9. W. K. Hayman, Meromorphic functions, Clarendon Press, Oxford, 1964.

10. On the characteristic of functions meromorphic in the plane and of their integrals, Proc. London Math. Soc. (3) 14A (1965), 93-128.

11. _ Research problems in function theory, Athlone Press, London, 1967.

12. _ Value distribution and A. P. gaps, J. London Math. Soc. (2) 28 (1983), 327-338.

13. R. Nevanlinna, Le théorème de Picard-Borel et la théorème des fonctions meromorphes, Gauthier-Villars, Paris, 1929.

14. G. S. Prokopovich, Fixpoints of meromorphic functions, Ukrain. Mat. Zh. 25 (1973), 248260; English transl.,Ukrainian Math. J. pp. 198-208.

15. P. C. Rosenbloom, The fixpoints of entire functions, Medd. Lunds Univ. Mat. Sem. [Tome Suppl.] (1952), 187-192.

16. G. Valiron, Lectures on the general theory of integral functions, Edonard Privat, Toulouse, 1923.

17. L. Yang, New researches on value distribution theory, Science Press, 1982. (Chinese)

Institute of Mathematics, Academia Sinica, Beijing, China China

Current address: Department of Applied Mathematics, Tsing Hua University, 100084 Beijing,

Department of Mathematics, The Hong Kong University of Science and Technology, KowloON, HoNG Kong 